\title{
Presenting Solutions to Increase Simultaneous Call in VOIP System by SIP Protocol - Based Media Server
}

\author{
Sajad Gharaguozloo ${ }^{1}$, Abdolhamid Zahedi ${ }^{2}$, Mohammad Norouzi ${ }^{2}$, Hamid Chegini ${ }^{2,}$ " \\ ${ }^{1}$ Telecommunication, of Non-profit Institution of Higher Education, ABA, Abyek, Qazvin, Iran \\ ${ }^{2}$ Non-profit Institution of Higher Education, ABA, Abyek, Qazvin, Iran \\ Email address: \\ sajadgharegozloo@yahoo.com (S. Ghareguozloo),H.zahedi.62@gmail.com (A. Zahedi),noroozi.62@gmail.com (M. Norouzi), \\ fmirzaei_91@yahoo.com (H. Chegini) \\ ${ }^{*}$ Corresponding author
}

\section{To cite this article:}

Sajad Gharaguozloo, Abdolhamid Zahedi, Mohammad Norouzi, Hamid Chegini. Presenting Solutions to Increase Simultaneous Call in VOIP System by SIP Protocol - Based Media Server. International Journal of Wireless Communications and Mobile Computing.

Vol. 4, No. 2, 2016, pp. 12-17. doi: 10.11648/j.wcmc.20160402.11

Received: February 21, 2016; Accepted: March 1, 2016; Published: March 12, 2016

\begin{abstract}
By development of multi-media in networks, the borders among networks are changed and all networks are approaching to be united. Unity of data, video and voice networks in one network has many advantages and disadvantages for users and servers. One of the disadvantages is unwanted events in network including load increase, jitter, information packet loss, delay and etc. and all these lead into low quality of voice and disconnection during simultaneous call. The use of multimedia server is one of the efficient ways to improve VOIP. We can enable the video conferences to transit information packets by media servers, so we can say: media servers can be used as core component for VOIP. In this research work, assessing of media servers is done by simulators that they produce RTP's connections, in additions as an experimental components SEMS that it's a source of media server, is used for asserting the quality by doing packets information with SIP. We can observe that the more increase of connections and pass of the certain threshold, the less of quality. In addition, the other performance metrics such as error rate And packet lost are asserted. The identification of load saturation points and the efforts to eliminate disturbing factors during the increase of simultaneous call in this telephone system, can present quality-based approach to servers of these networks.
\end{abstract}

Keywords: VOIP, SEMS, SIP, Increase of Simultaneous Call

\section{Introduction}

Today, with the technology progress and creating data networks (e.g. internet) and using these networks for voice transmission, a new method is crated in telecommunication connection and it is called VOIP technology or "Voice. Over Internet Protocol" and the networks based on this technology are called VOIP networks.

Due to various facilities providing for users, these networks are welcomed as they are considered as the alternative of current telecommunication networks.

Any new technology has some limitations. One of the problems of data-based networks is the problem of increase of simultaneous call in them. This study attempts to evaluate that in a $\mathrm{SIP}^{1}$-based VOIP telephone system, by increasing

${ }^{1}$ Session Initiation Protocol participants in a voice conference simultaneously, media server behavior is changed and then by the results of experiment and experience, proposed solutions to increase calls in this telephone system as network can be defined. In the test, open source media service as called $\mathrm{SEMS}^{2}$ is applied. The results of test can be used to increase call in each network-based telephone system.

Regarding the evaluation of media service performance, there are various researches as follows:

In reference [1], a hash table is used to test the trend of SIP on virtual media server. In this study by a SIP proxy, about 5 thousands calls were established with different times and after the evaluation of outputs, it was shown that by the increase of calls about 300 calls per seconds, due to losing signal, unsuccessful call rates were increased. 
Based on hash table system, this trend is improved and is initiated from 300 to 500 calls per seconds.

In reference [2], two parts of SIP software as one server and another one customer is used to send signaling traffic and the rate of calls is increased from 5 calls to 60 calls simultaneously per seconds and a call control system is considered to be used for terminating the remaining calls exceeding $3.5 \mathrm{~m}$, about $37 \%$ of calls are in time out and are finished before being terminated by controller [3].

The paper is structured as follows. The second section is dedicated to the introduction of VOIP telephone system and its advantages to old communication lines. Third section is about the evaluation of SIP protocol as the protocol playing the main role in creating calls. Fourth section introduces media server and voice conferences and constituent elements of a conference. Finally, fifth section is regarding the evaluation of media server and its test in terms of $\mathrm{RTP}^{3}$ load. Final section is about the results of discussions and solutions to increase simultaneous call in VOIP system by SIP protocol-based media.

\section{What Is VOIP}

VOIP is the technology providing data network for telephone conversations. By VOIP, human voice is sent via $\mathrm{IP}^{4}$ information packets and via data-based network as internet [4]. In other words, VOIP technology is a set of hardware and software enabling us to use data network as transition mediator for telephone calls [5].

\subsection{The Advantages of VOIP over PSTN}

In case of using PSTN ${ }^{5}$, users pay the time cost by the company responsible for PSTN line (telecommunication), more than one person is not communicated simultaneously [6]. In VOIP technology, we can talk simultaneously with more than one without any extra cost. Also data is exchanged during talking (e.g. image, chart and video images). VOIP service provides all services presented by ordinary phone [7] [8].

\subsection{VOIP Defects in Contrast with PSTN}

Because of connecting VOIP to the internet, invaders may be able to do something such as cut the connection, over hearing or interrupting in this service [9] [10].

Unlike old phone lines, when the electricity goes out, we cannot use VOIP. This causes some problems in security system in the houses and in that time, there is lack of access to emergency calls.

Also, as the internet's issues, perhaps we can't receive internet packets that they were sent. This problem causes some disadvantages in VOIP system.

\section{SIP Protocol}

Protocol means a set of rules determining the information

3Real Time Protocol

4Internet Protocol

5Public Switched Telephone Network exchange. VOIP system works on network and we need a communication language or protocol for data transmission.

SIP is an application-layer control (signaling) protocol for creating, modifying and terminating sessions with one or more participants. [11] [12] The invitations sent by SIP are used for sessions on IP network [13].

\subsection{Important Elements of SIP}

- User agent (UA) creates SIP transactions or responds alone.

- User agent client (UAC) creates SIP requests and accepts responses and reactions of SIP.

- User agent server (UAS) gets SIP requests and sends the responses.

\subsection{SIP Requests}

SIP requests are the messages being sent from customer to server to call for SIP operation and most important examples are as follows:

- INVATE or invitation: It is a method showing that invitation client is invited to participate in a network.

- $\mathrm{ACK}^{6}: \mathrm{ACK}$ request shows that a client agent of the last request has received an invitation. ACK is used continuously for 200 OKs.

- BYE: An UA uses BYE request to terminate old session. Connection by SIP is shown in Figure 1.

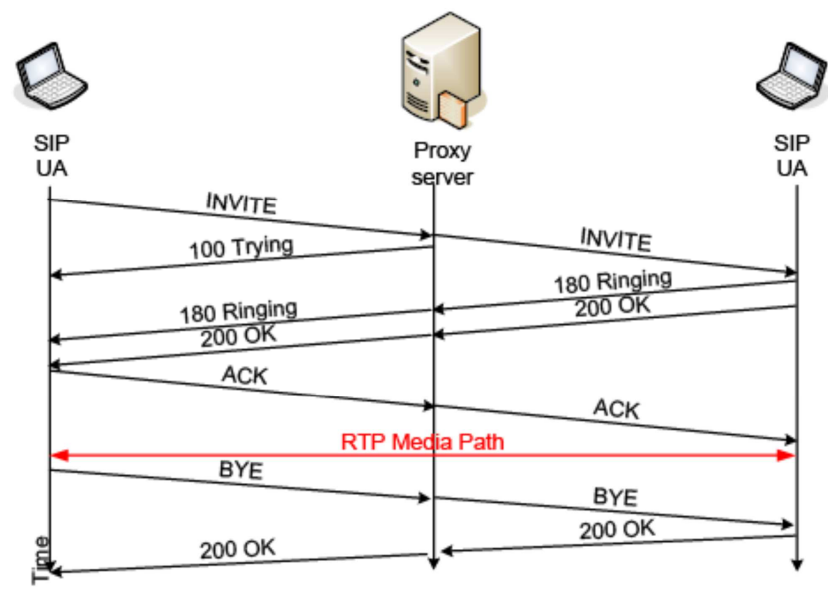

Figure 1. Connection by SIP.

For a two-party session, if a message is sent by user agent, the proxy sends two messages of Trying and Ringing to the user agent and if the invitation is accepted, a $200 \mathrm{OK}$ message is sent to user agent and user agent acknowledges the message of OK. RTP ${ }^{7}$ or voice and video stream can be exchanged among them. [14] If the user agent attempts to terminate the session, BYE message is sent to server proxy and to respond this request, by sending message acknowledges the termination of session.

6Acknowledge

7Real Time Protocol 


\section{The Role of Media Server in Voice Conference}

The history of media server was at the same time with the history of automatic clock and automatic teller in 1960. In 1990, a great revolution was occurred in independent message transmission from fax and voice conference servers and in 1998-1999, media servers were similar to new forms.

The example of the performance of media server is conference services. These services by combining voice streams send it for all participants in conference.

\subsection{Constituents of Voice Conferences}

Generally, connection sessions with multiple participants are recognized as conference [15]. A voice conference is composed of conference server and participants. Conference server is composed of a focus and a mixer. Focus is in control of conference and mixer attempts to transfer multi-media RTP stream among participants [16].

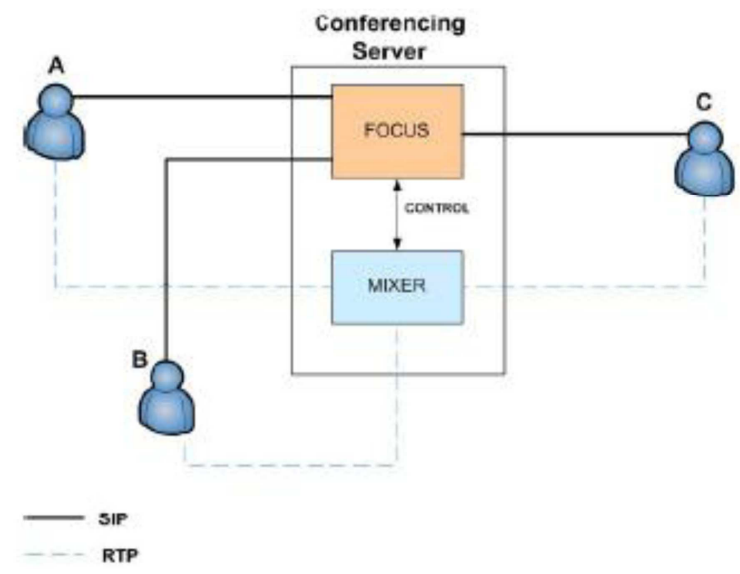

Figure 2. Diagram of a voice conference.

Mixer receives control orders from focus unit. Voice streams are received by mixer from participants and streams are distributed among them again by mixer. Each participant receives the sum of media streams of other participants minus RTP stream.

\subsection{SEMS Media Server}

The main duty of a media server is processing mass media stream.

The following Figure shows the architecture of a simple media server.

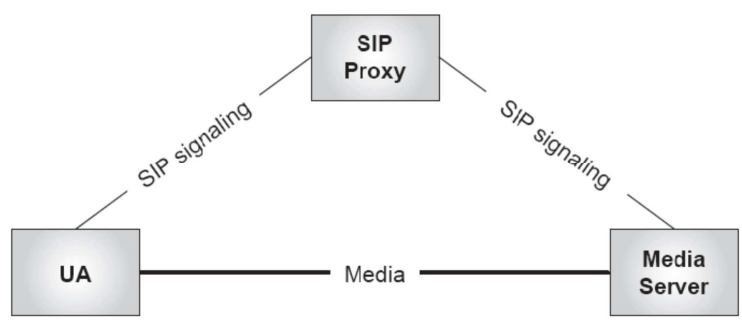

Figure 3. Architecture of a media server.
Some of the most important duties of a media server are as follows:

- Combining media stream in conference and its re-sending to participants

- Conversion of text to speech

- Automatic detection of voice

SEMS media server [17] in VOIP services based on SIP can be used. SEMS has an internal focus acting as its control sector.

\section{A Method to Evaluate Media Server and Presenting Solution to Increase Simultaneous Calls in It}

In this paper, we evaluate SEMS media server and use the conference service of this media server.

The following Table expresses the test conditions. To test this test, encoder-decoder G711 as free and standard is used.

Table 1. Test conditions Table.

\begin{tabular}{ll}
\hline Explanation & Type \\
\hline Pentium IV- 1.86GHZ / 3096MB & System \\
Pentium IV- 1.86GHZ / 3096MB & Directing system \\
Pentium IV- 1.86GHZ / 3096MB & Traffic transmitter \\
Pentium IV- $1.86 \mathrm{GHZ} / 3096 \mathrm{MB}$ & Media server \\
\hline
\end{tabular}

Figure 4 shows the location of media server and SIP in test.

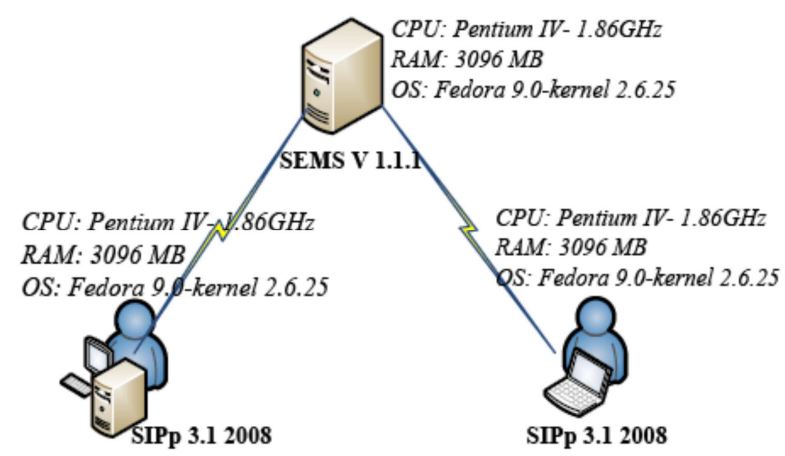

Figure 4. The location of media server and participants in test.

All tests as explained later are performed under equal conditions and after sending background load or warming media server. It is worth to mention that in these tests, rate of 10 calls per seconds is considered and 40 seconds RTP on server is sent. In the first test in this study, the aim of study is to test load, we want to evaluate what happens to $\mathrm{CPU}^{8}$ load by increasing the number of participants in conference?

As shown, when the number of participants in conference is more than 190, the processer load reaches $100 \%$ and the number of participants in this conference shouldn't be more than 75 and the load is not above $80 \%$. CPU load can be dependent upon applied hardware and type of directing system.

In this study, we try to investigate the cause of losing

${ }^{8}$ Central Personal Unit 
packets and jitter in this system.

Generally, the reasons of packets loss, signal reduction in network, saturation of communication links, defect hardware and the method of routing in routers. As the media server has limited memory and limited processing resources, by increasing the number of participants in server conference, it reaches saturation level and the percent of packets loss is increased. As shown in the following Figure, if the number of participants exceeds 75, packets loss is increased as sudden but the number of participants achieves 75 , the percent of packets loss is about 3\% as acceptable for an encoder-decoder
G 711 acceptable. In some papers [18], quality of maximum value service is by one percent.

By packets loss, we can expect jitter phenomenon.

One of the most important indices in evaluation of VOIP service quality is jitter. Jitter in network can be due to traffic, change of routing and even bad configuration of device. In this test, as server capacity is limited, by increasing the number of callers, disturbance is made between media streams combination with their re-distribution among participants and jitter is occurred.

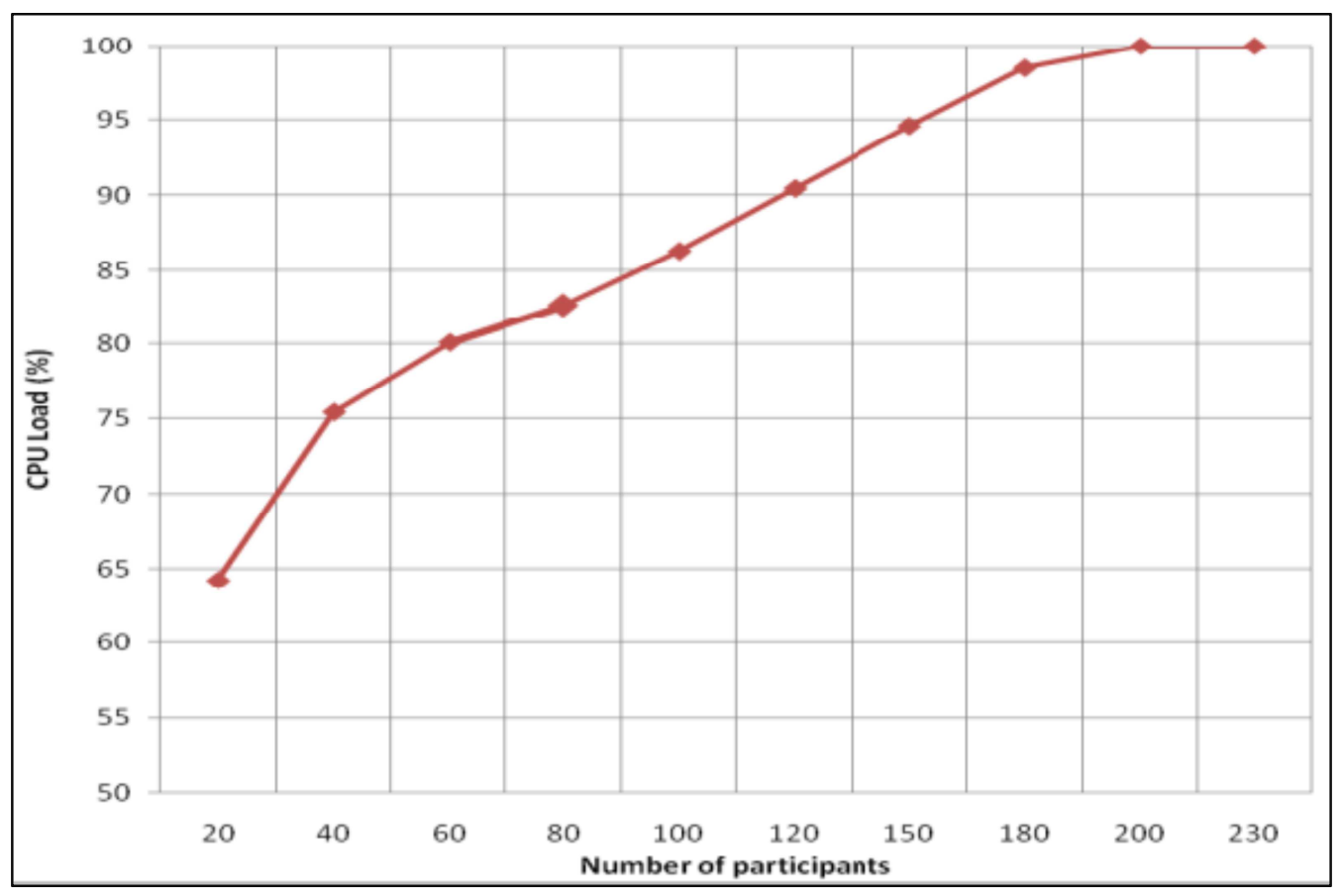

Figure 5. CPU load chart by increasing the number of participants.

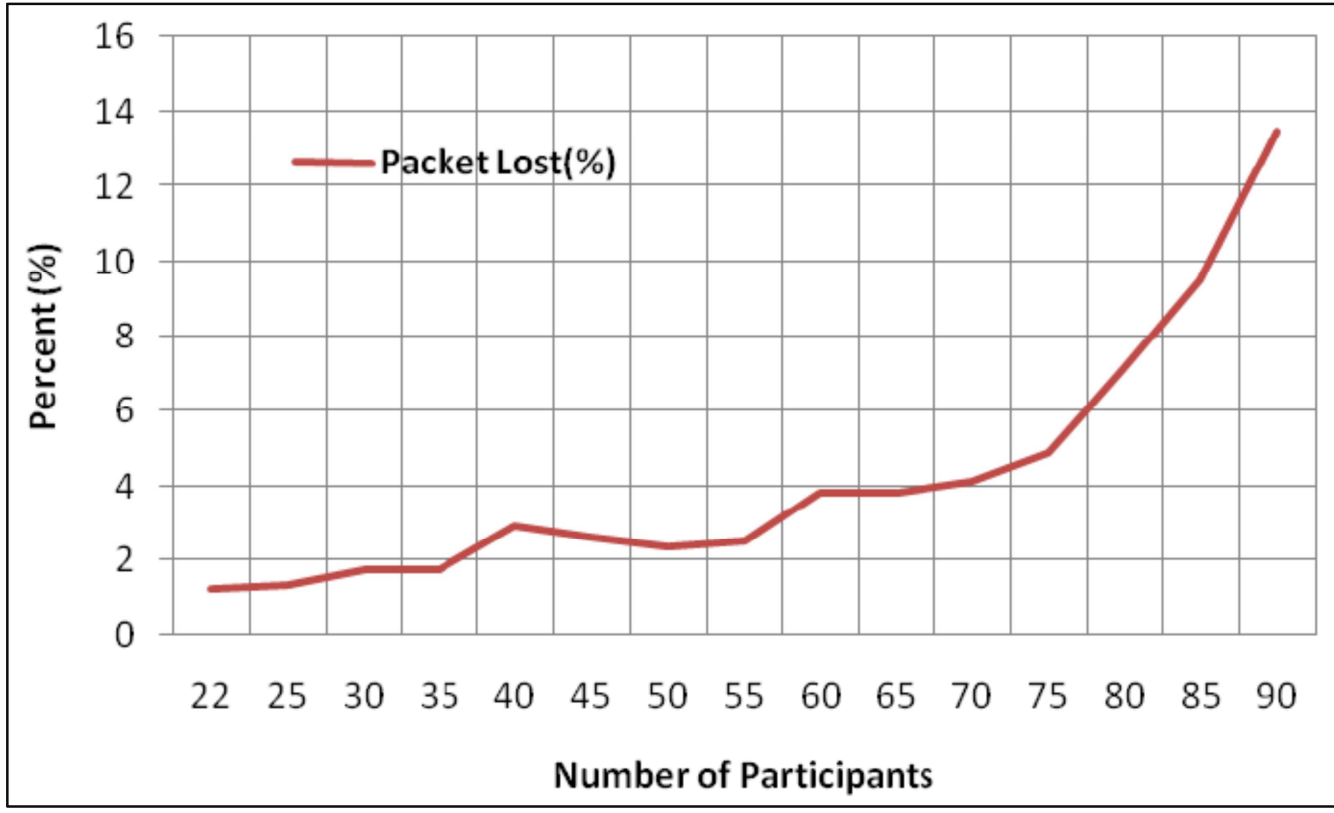

Figure 6. The chart of number of lost information packets by increasing the number of participants. 


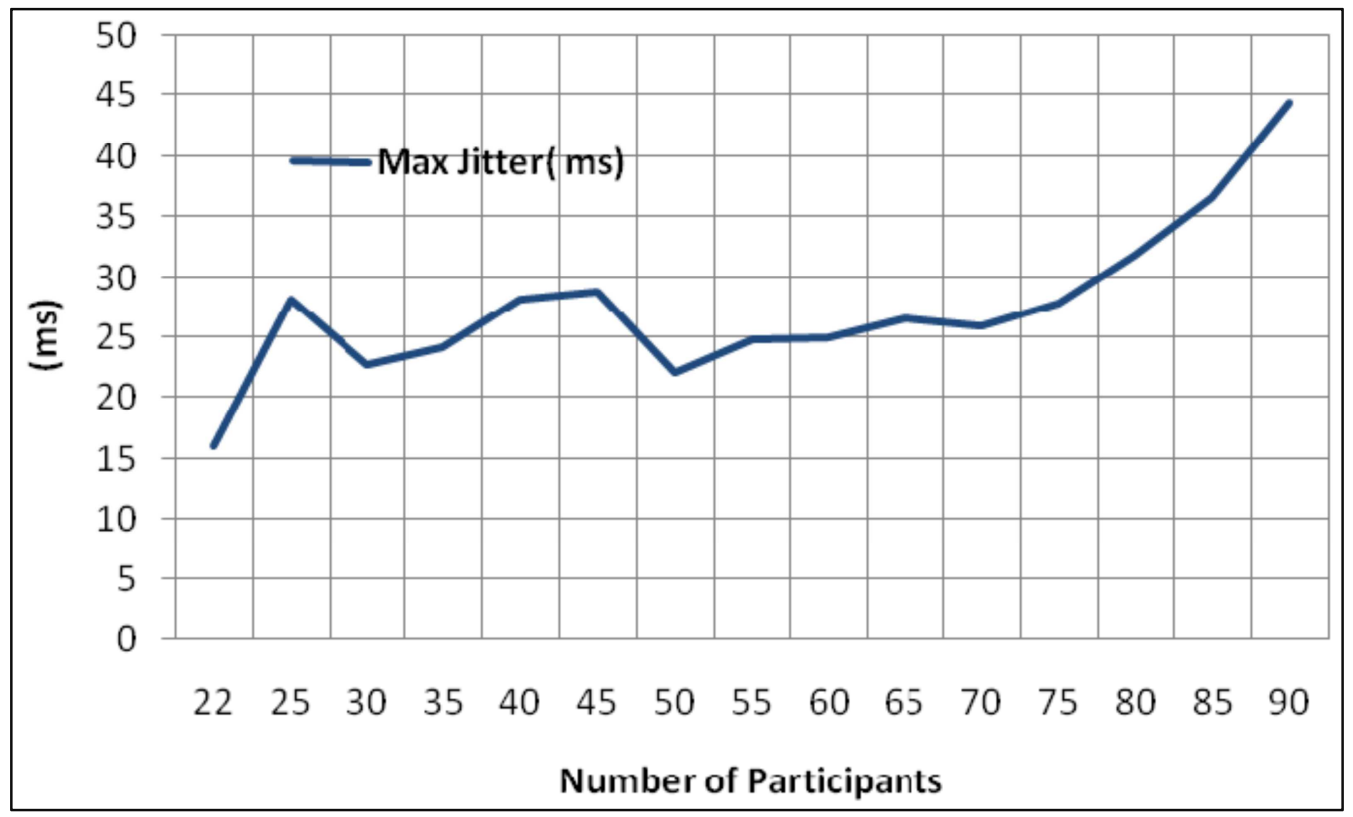

Figure 7. The chart of jitter by increasing the number of calls.

\section{Conclusion}

In this study, it was shown that by increasing the number of participants in a conference, media server is saturated after a definite point and no new participant is accepted. The main goal of simultaneous calls is quality. Thus, evaluation of RTP loan on media server can present service-quality based attitude but at signaling level, it is only zero and one and we can be aware of making or not making calls (number of calls).

It is observed that after passing saturation point, connection is established in media but lack of quality avoids continuing work.

Thus, based on the test and experiences, we can use the following methods to achieve the increase of number of simultaneous calls in a VOIP system:

- Increasing the capacity of servers by combining two or some servers as the number of callers is increased and no problem is made in combination and distribution of media streams.

- In writing programming for VOIP telephone system, we should try to have an optimal application. The main purpose of efficient plan is only support your needs of one call or conference call in VOIP.

- During telephone conference, if the number of callers is high, recording conversation is avoided to prevent server to be involved other affairs.

- For the entire system, only a protocol is used, for example applied protocols in receiver and transmitter are both SIP. Use of different protocols in transmitter and receiver causes spending more time to exchange their language to each other and the function of system is decreasing.

- Codecs of voice transmission are similar to make the application as optimal. As codecs of voice transmission aren't same, it causes waste of time and waste of some calls when at the same time the calls increase.

- We shouldn't use compressing Codecs of voice transmission as these Codecs reduce bandwidth, they reduce quality in information packets and number of callers are similar at a time.

Knowing the methods of increasing the number of simultaneous calls in VOIP systems and reaching saturation points and acquiring them can be of great importance for VOIP servers.

\section{References}

[1] H. Wook, S. Kang, D. Kim, "Performance Enhancement of SIP proxy server by using Ihash for matching transaction", IEEE, ISBN 978-89-5519-131-8 93560, Feb 2007

[2] Mauro Femminella, Roberto Francescangeli, Francesco Giacinti, Emanuele Maccherani, "Design, Implementation, and performance of an advanced SIP-based call control for VoIP services", IEEE, ISBN 978-1-4244-3435, 2009

[3] http://sipp.sourceforge.net/doc/reference.html

[4] Montoro, P, Casilari, E, 2009, A Comparative Study of VoIP Standards with Asterisk, Fourth International Conference on Digital Telecommunications

[5] Iseki, F, Sato, Y, Kim, M. 2011, VoIP System based on Asterisk forEnterprise Network, ICACT2011

[6] Pantelis A. Frangoudisa, George C. Polyzosb, On the performance of secure user-centric VoIP communication, 2014, Computer Networks Volume 70, 9 September Pages 330-344

[7] Abdul Qadeer, M, Shah, K, Goel, U, 2012, Voice - Video Communication on Mobile Phonesand PCs' using Asterisk $E P B X$, International Conference on Communication Systems and Network Technologies

[8] WWW.VOIP-IRAN.COM 
[9] Nikos Vrakasa, Dimitris Geneiatakisb, Costas Lambrinoudakisa, Obscuring users' identity in VoIP/IMS environme, 2014, Computers \& Security, Volume 43, June, Pages 145-158

[10] Ryan Farley, Xinyuan Wang, Exploiting VoIP softphone vulnerabilities to disable host computers: Attacks and mitigation, 2014, International Journal of Critical Infrastructure Protection, Volume 7, Issue 3, September, Pages $141-154$

[11] Liping Zhang, Shanyu Tang, Shaohui Zhu, An energy efficient authenticated key agreement protocol for SIP-based green VoIP networks, 2016, Journal of Network and Computer Applications, Volume 59, January, Pages 126-133

[12] Jinzhu Wanga, et al, Probe-based end-to-end overload control for networks of SIP servers, 2014, Journal of Network and Computer Applications, Volume 41, May, Pages 114-125

[13] Basicevic, M. Popovic, D. Kukolj, 2008, Comparison of SIP and H. 323 Protocols, Proc. of The Third International Conference on Digital Telecommunications (ICDT'08), Bucharest (Romania), Jul. pp.162-167

[14] Regis J. (Bud) Bates, Chapter 6 - Other protocols SRTP, ZRTP, and SIPS, 2015, Securing VOIP Keeping your VOIP Network Safe, Pages 123-150
[15] J. Rosenberg, H. Schulzrinne, G. Camarillo, A. Johnston, J. Peterson, R. Sparks, M. Handley, E. Schooler, "SIP: Session Initiation Protocol", RFC 3261, June 2002

[16] J. Rosenberg, "A Framework for Conferencing with the Session Initiation Protocol (SIP)", RFC 4353, February 2006

[17] R. Even, N. Ismail, "Conferencing Scenarios", RFC 4597, August 2006

[18] http://www.iptel.org/sems.

[19] C. Partridge, "Isochronous Applications Do Not Require Jitter-Controlled Networks", RFC 1257, September 1991

[20] H. Schulzrinne, S. Casner, R. Frederick, V. Jacobson, "RTP: A Transport Protocol for Real-Time Applications", RFC 3550, July 2003

[21] A. H. Ashouri, F. Samsami, A. Akbari, "E-Learning Media Server Evaluation and its architecture modeling with signaling load tests," ICeLT, IUST, Tehran, Iran, Dec 2009

[22] A. H. Ashouri. "Media Server Evaluation and Real-Time Tests" Iran University of Science and Technology, B. Sc Thesis, p46-61, Sep 2009 